\title{
Quench energy studies in ITER conductors for different magnetic field perturbations with Jackpot and THEA combined models
}

\author{
T. Bagni ${ }^{1,2}$, J. L. Duchateau ${ }^{3}$, A. Devred $^{4}$ and A. Nijhuis $^{1}$ \\ ${ }^{I}$ University of Twente, Faculty of Science \& Technology, 7522 NB Enschede, The Netherlands \\ ${ }^{2}$ Ghent University, Department of Applied Physics, B-9000 Gent, Belgium \\ ${ }^{3}$ CEA/DRF/IRFM,CEA Cadarache 13108 St Paul-lez-Durance, France \\ ${ }^{4}$ CERN, Technology Department, Geneva, Switzerland
}

The electromagnetic-thermal models for Cable-in-Conduit Conductors JackPot-ACDC and THEA (Thermal, Hydraulic and Electric Analysis of superconducting cables) are combined predicting the stability of ITER Central Solenoid conductors. The combination of both models allows the prediction of the effect of any type of magnetic field perturbation in time, relevant for the magnet coils during the plasma operation scenario of the reactor. At present, there is no experiment for testing the stability of the ITER $\mathrm{Nb}_{3} \mathrm{Sn}$ conductors under such conditions. Only limited experimental data on Minimum Quench Energy (MQE), defining the conductor stability, are available but the time and magnetic field amplitude settings are completely different from the actual ITER operating conditions. Nevertheless, such tests are useful as a basis to calibrate and benchmark the codes. The JackPot-THEA combination allows to determine the MQE for any magnetic field change in time and to fully describe the involved electromagnetic phenomena in strand-level detail in terms of local power dissipation and (peak) electric field along all strands. Thermally, the computation is still on a global scale for identifying the quench initiation and propagation. The predictions from the combined codes are in good agreement with the experimental results and provide a solid basis for extrapolative scaling of CICC's stability under plasma operating conditions.

Disclaimer: The views and opinions expressed herein do not necessarily reflect those of the ITER Organization.

\section{Introduction}

The ITER magnet system is subjected to fast changes in currents and magnetic fields during the plasma-operating scenario. The most critical factor concerning the level of magnetic field rates is the Central Solenoid (CS), which can decrease the magnetic field from $13 \mathrm{~T}$ with a maximum rate of 1.5 $\mathrm{T} / \mathrm{s}$ during plasma initiation. This extreme condition is not easily reproducible in existing experimental facilities. The only experimental data available for quantitative analysis are produced at the ITER Central Solenoid Model Coil (Naka, Japan) [1] and SULTAN conductor testing facility (Swiss Plasma Centre) [2], but conductor layout, time and magnetic field amplitude settings are different from the ITER operating conditions; particularly for SULTAN, the testing conditions for quenching do not cover the operating conditions. However, the test results of the ITER conductor qualification program [3] are very useful for calibrating the codes used in the analysis presented here.

To achieve accurate transient stability predictions and to study the behavior on the strand level for Cable-in-Conduit Conductors, a code must calculate AC losses with high accuracy and manage variations in strand currents, magnetic field amplitude and temperature. JackPot-AC/DC [4] [5] [6] is a suitable code for calculating local ohmic losses since it can generate the geometry of a real CICC with strand level precision and simulate the electromagnetic behavior of a conductor during stability tests and a Plasma Scenario (PS). JackPot also contains a thermal routine, which can calculate the average temperature in the cable petals. However, it is not able to model a quench and its propagation along the conductor. To add this feature, the THEA code [7] (Cryosoft [8]) is used. The two codes 
cannot function together, but it is possible to create special routines that allow them to communicate. The resulting system, called JackPot-THEA in this paper, can predict the stability limit of the analyzed cable, starting only from testing boundary conditions of current, temperature and magnetic field. The calibration and the validation of the code system have already been presented in a previous study [9].

The analysis conducted in this study aims to better understand the stability behavior of CS conductors under operating conditions. We start with the results based on the SULTAN short sample stability tests, with very short pulse duration $\left(\mathrm{t}_{\text {pulse }}=128 \mathrm{~ms}\right.$ ) for verification of the model, and then analyze different perturbation times with longer pulse duration. The most severe condition for the CS coils during the 15MA PS is during the plasma initiation for about 1-2 s [10], while the SULTAN singular sine wave pulse period is only $0.128 \mathrm{~s}$. Although with cautiousness, the stability results obtained from the analysis of the SULTAN tests are used for extrapolation with JackPot-THEA investigating the $\mathrm{MQE}$ for more relevant magnetic field disturbances by taking 1 and $5 \mathrm{~s}$ single sinusoidal pulses.

The different time scale and amplitude of the pulses affect the current and temperature distribution diffusion process, which may be different from that of the fast SULTAN pulse. Both peak electric field and temperature profile are investigated to determine an AC quench criterion applicable as threshold for the ITER plasma scenario.

\section{Experimental stability tests}

The stability tests used to benchmark the models are performed in the SULTAN facility. The experimental set up used in SULTAN to test the ITER CS conductors consists of a conductor sample assembled in U shape configuration [11] inserted in a magnet system able to generate orthogonal DC and AC magnetic fields [2]. The conductor central channel is closed so that the helium can flow only in the cable bundle, with controlled mass flow rate in the range $1-10 \mathrm{~g} / \mathrm{s}$. The power dissipation generated in the cable by the AC field is indirectly measured using a calorimetric method. The helium temperature increase, measured using temperature sensors located at the boundaries of the AC field region, is integrated using the helium mass-flow and specific heat, obtaining the enthalpy change in the conductor [12].

The stability tests are carried out in presence of transport current and background magnet field. The power loss is induced in the conductor using a fast single sinusoidal magnetic field pulse with the AC coil. The pulse amplitude is increased stepwise until a quench occurs. The ITER conductor sample used in this study is referred to as CSJA8, the conductor parameters are listed in Table 1.

Table 1 - Parameters of the ITER CSJA8 sample, tested in SULTAN

\begin{tabular}{l|l}
\hline \multicolumn{2}{l}{ CSJA8 } \\
\hline Shape & Circle-In-Square \\
Main outer dimension [mm] & $49.0 \times 49.0$ \\
Jacket inner diameter [mm] & 32.6 \\
$\begin{array}{l}\text { Central spiral inner and outer diameter } \\
\text { [mm] }\end{array}$ & $7-9$ \\
$\begin{array}{l}\text { Cable layout sub-stages } \\
\text { Cable layout twist pitches [mm] }\end{array}$ & $(2 \mathrm{sc}+1 \mathrm{Cu}) \times 3 \times 4 \times 4 \times 6$ \\
\hline
\end{tabular}


Jacket material

Void fraction [\%]

Joint layout

Strand manufacturer

Strand Cu:nonCu

RRR
JK2LB

33.6

Solder Filling

KAT

1.0

$>100$

The strand critical current is modeled using the ITER scaling law for $\mathrm{Nb}_{3} \mathrm{Sn}$ wire [13]. The parameters used to model the KAT strand [14] were measured at the University of Twente, and summarized in Table 2. The only unknown free parameter required for modeling the strand critical current of the sample is the axial strain applied to the strands $\mathrm{Nb}_{3} \mathrm{Sn}$ filaments due to the cooling down and electromagnetic charging process [15]. The current sharing temperature $\left(T_{c s}\right)$ measurements are used to determine the axial strain parameter. The $T_{c s}$ is defined as the temperature where the electric field reaches $E_{c}=10 \mu \mathrm{V} / \mathrm{m}$ [16]. Knowing the measured $T_{c s}$ and the test conditions in terms of current and applied magnetic field, the $\mathrm{Nb}_{3} \mathrm{Sn}$ scaling law can be used to calculate the effective axial strain. The electromagnetic forces applied on the conductor influence the effective strain. Therefore, the $T_{c s}$ testing conditions in terms of magnetic field and transport current play an important role in determining the effective strain. In SULTAN, just before the stability test is initiated, $T_{c s}$ is measured using the same test conditions as those applied during the test, i.e. performed at $B_{d c}=9 \mathrm{~T}$ and $I_{o p}=40$ $\mathrm{kA}$. This $T_{c s}$ measurement allows to calculate the effective strain necessary to model the MQE tests, in this case $\varepsilon_{e f f}=-0.54 \%$.

Table 2 - KAT Nb${ }_{3} \mathrm{Sn}$ strand parameters for $J c(B, T, \varepsilon)$ characterization.

\begin{tabular}{l|l}
\hline \multicolumn{1}{c}{ KAT strand } \\
\hline$C_{a 1}$ & 45.33 \\
$C_{a 2}$ & 0.000 \\
$\varepsilon_{0 a}$ & $0.325 \%$ \\
$\varepsilon_{m}$ & $-0.164 \%$ \\
$B_{c 2 m(0)}$ & 29.26 \\
$T_{c m}$ & 16.17 \\
$C$ & 12822 \\
$p$ & 0.419 \\
$q$ & 1.431 \\
\hline
\end{tabular}

A typical plot of the results from MQE tests is shown in Figure 1. The energy deposited trough the applied AC field per unit volume of composite is shown as a function of the pulsed field maximum amplitude, $B_{a}$. Because of the self-heat generated by the quench avalanche-like process, the actual quench energy (full dots) can only be extrapolated from the energies generated by the previous smaller pulses. The extrapolation is performed using a linear fit, because there are only few measured energies for each temperature margin (usually two), therefore, it is not possible to define a better fit. The figure shows the impact of the operating temperature on the MQE at a transport current of $40 \mathrm{kA}$. The higher the operating temperature, the lower is the deposited energy necessary to initiate a quench, 
and consequently the magnitude of the magnetic field pulse. The quench energy calculated at $T=8.6$ $\mathrm{K}$ downstream the High Field Zone (HFZ) is higher than the one at $\mathrm{T}=8.4 \mathrm{~K}$, which is probably due to the unstable helium temperature fluctuations. The helium temperature in the SULTAN setup becomes unstable at higher values of temperature, resulting in a less accurate assessment of the quench energy.

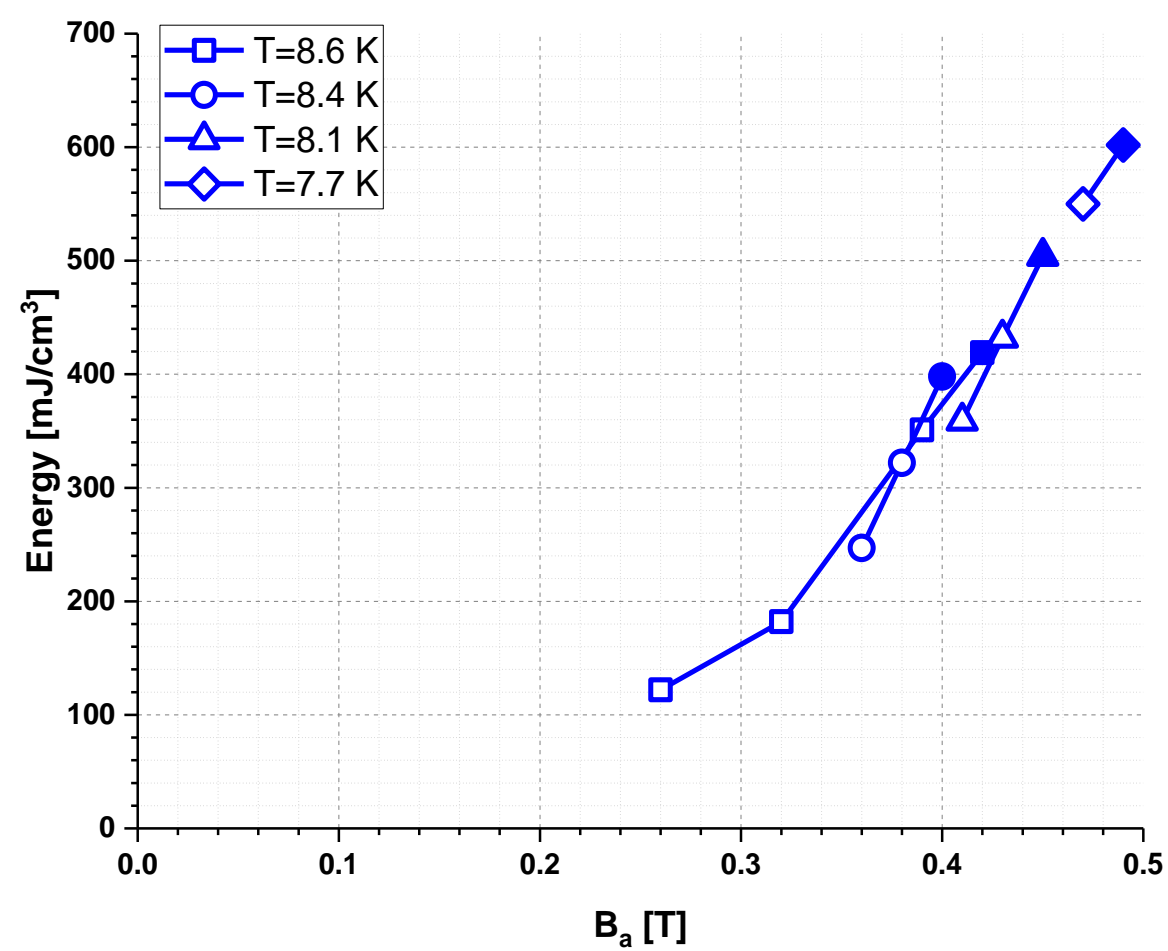

Figure 1 - Experimental stability test results of the CSJA8 sample with a transport current of $40 \mathrm{kA}$ and pulse period of $0.128 \mathrm{~s}$. The value $B_{a}$ represents the peak magnetic field applied on the conductor during the fast field pulse used to generate a quench. The filled symbols indicate the extrapolated energy levels where the cable is quenching.

\section{JackPot-THEA model}

THEA is a 1-D model, used to analyze hydraulic and thermal behavior of superconducting wires and cables. The sample conductor is defined by using hydraulic and material parameters, summarized in Table 3. THEA models a simplified conductor $1.5 \mathrm{~m}$ long, divided in two thermal and one hydraulic component. One thermal component represents $\mathrm{Nb}_{3} \mathrm{Sn}$ and segregated copper strands while the second component is the stainless steel jacket. The hydraulic component is the supercritical helium that flows in the cable bundle.

JackPot AC/DC is a numerical 3-D model, able to reproduce a full CICC as a network of superconducting and resistive elements describing the trajectories of all the strands inside the cable. The discretization used to model the conductor is a fraction of the length of the first twist pitch, usually $d z<1 / 5 L p_{1}$ [6]. Therefore, usually $d z$ is from 2 to $5 \mathrm{~mm}$ depending on the cable twist pitch pattern. The contacts among the strands are about $10^{4}-10^{5}$ generating a consequent huge number of current loops that is difficult to evaluate. The value of the inter-strand contact resistance is the only free parameter in the model. The contact resistance is determined by fitting the CSJA8 AC loss measurements with JackPot simulated AC loss. After the calibration, JackPot is able to successfully 
calculate the power losses generated in the cable for every magnetic field perturbation. By increasing the initial temperature of the simulation and approaching the current sharing temperature $\left(T_{c s}\right)$, the strands start to saturate. The strand $I_{c}$ saturation is taken into account for computation of the coupling loss at high temperature and magnetic field. The current distribution among the strands including the transport and coupling currents in the whole cable is considered for all strands [6]. The electric field is locally calculated along all strands of the cable. This means that saturation is implicitly taken into account since reaching the superconducting transition for a part of the strands (and particular sections of strands depending on their location) will limit the current. At the same time it will force other strands, which are not saturated, to take more current. This means that the saturation of coupling loss is fully taken into account, just as all shielding effects from all current loops of individually connected strands in the cable.

With THEA, using the output results from JackPot, the thermal process evolution in the conductor can be predicted. The heating zone (HZ) of $0.39 \mathrm{~m}$ long is the effective length of the AC field generated in SULTAN. The HZ is placed in the center of the $1.5 \mathrm{~m}$ cable modeled with THEA.

Using the combined models, it is possible to predict the MQE for the CSJA8 using the same procedure as in the SULTAN experiment [9]. In JackPot-THEA the transport current and background field are set constant while the magnetic field pulse is progressively increased up to the quench in the conductor.

Table 3 -Hydraulic and thermal parameters of the CSJA8 sample used during the stability calculations performed with JackPot + THEA [17].

\begin{tabular}{l|l}
\hline Hydraulic parameters & CSJA8 \\
\hline$A_{\text {Helium in annulus }\left[\mathrm{mm}^{2}\right]}$ & 259 \\
Wetted perimeter bundle $[\mathrm{mm}]$ & 2558 \\
Wetted perimeter jacket $[\mathrm{mm}]$ & 103 \\
Void fraction $[\%]$ & 33.6 \\
Mass flow $[\mathrm{g} / \mathrm{s}]$ & 3.3 \\
Thermal parameters & \\
$A_{S c}\left[\mathrm{~mm}^{2}\right]$ & 161 \\
$A_{\text {copper }}\left[\mathrm{mm}^{2}\right]$ & 321 \\
$A_{\text {bundle }}\left[\mathrm{mm}^{2}\right]$ & 482 \\
$A_{\text {jacket }}\left[\mathrm{mm}^{2}\right]$ & 1566 \\
\hline
\end{tabular}

The validation of the JackPot and THEA model combination is reported in [9]. The SULTAN stability test is compared with the JackPot-THEA calculated energies, see Figure 2. The JackPot-THEA energy is comparable with the SULTAN stability test results. The JackPot-THEA energies are slightly conservative, for the same applied magnetic field JackPot generates about 10\% higher energy deposition. The result of the JackPot-THEA simulation is independent from the experimentally determined MQE, the only information needed to simulate the stability tests are the coupling loss, the $T_{c s}$ measurements, the geometry of the conductor and the scaling law parameters of the strand used in the cable.

The testing boundary conditions used for the stability simulations are summarized in Table 4 . In the following paragraphs, the simulation results for $1 \mathrm{~s}$ and $5 \mathrm{~s}$ single sinusoidal magnetic field pulse period will be analyzed and compared with the $0.128 \mathrm{~s}$ pulse period results from [9]. The sine wave is chosen in order to minimize the difference between the model and the SULTAN fast pulse experiment 
and to clarify the influence of the pulse period and amplitude. Fields variations relevant for Plasma Operating conditions can be applied later on. The $5 \mathrm{~s}$ period analyses is also used to investigate the impact of the central channel on cable stability.

Table 4 - Testing conditions of the stability tests simulated with JackPot-THEA

\begin{tabular}{l|l}
\hline & CSJA8 \\
\hline Background Field [T] & 9 \\
Current [kA] & 40 \\
Temperatures [K] & $7.7-8.6$ \\
Sinusoidal pulse period [s] & $0.128-1-5$ \\
\hline
\end{tabular}

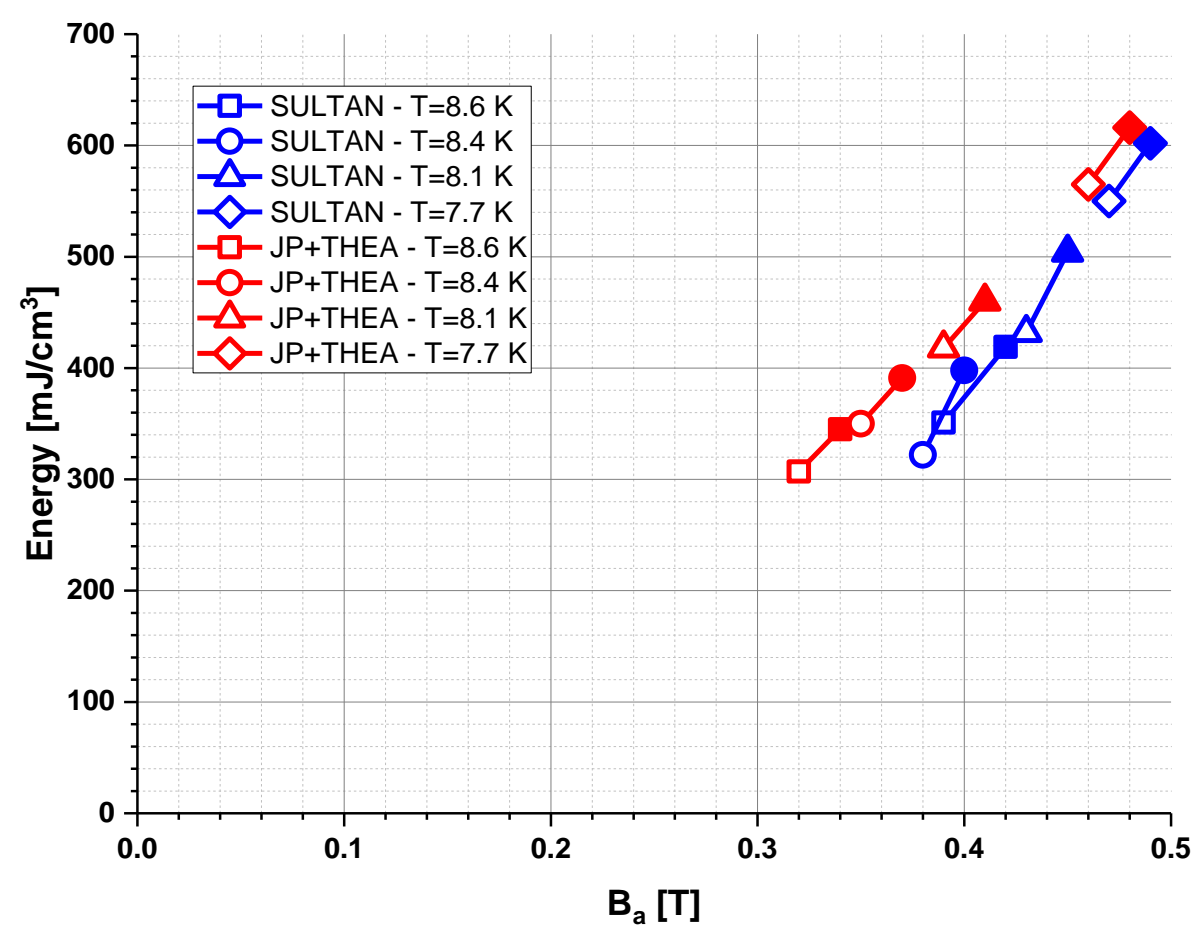

Figure 2 - Results of the CSJA8 conductor with a transport current of $40 \mathrm{kA}$ and a magnetic field pulse period of $0.128 \mathrm{~s}$. The energy, measured in Sultan and calculated with JackPot-THEA, is shown as a function of the magnetic field pulse amplitude, $B_{a}$. The open dots indicate recovery while full dots represent a quench.

\section{Stability for $1 \mathrm{~s}$ pulse period}

The electromagnetic and thermal testing conditions are the same as used to model the SULTAN MQE tests, but the single sine wave pulse period is set to $t=1 \mathrm{~s}$. For longer pulse period it is necessary to increase the magnetic field pulse amplitude in order to initiate a quench. The magnetic field amplitude is selected in the range $B_{\mathrm{a}}$ from $0.5 \mathrm{~T}$ to $1.2 \mathrm{~T}$. The predicted results of the MQE simulations are shown in Figure 3, together with the results obtained for the pulse time period of $0.128 \mathrm{~s}$. Increasing the single sinusoid pulse period from $t=0.128 \mathrm{~s}$ to $1 \mathrm{~s}$ by a factor $\sim 7.8$, increases the MQE in the cable by a factor 2.3 . 


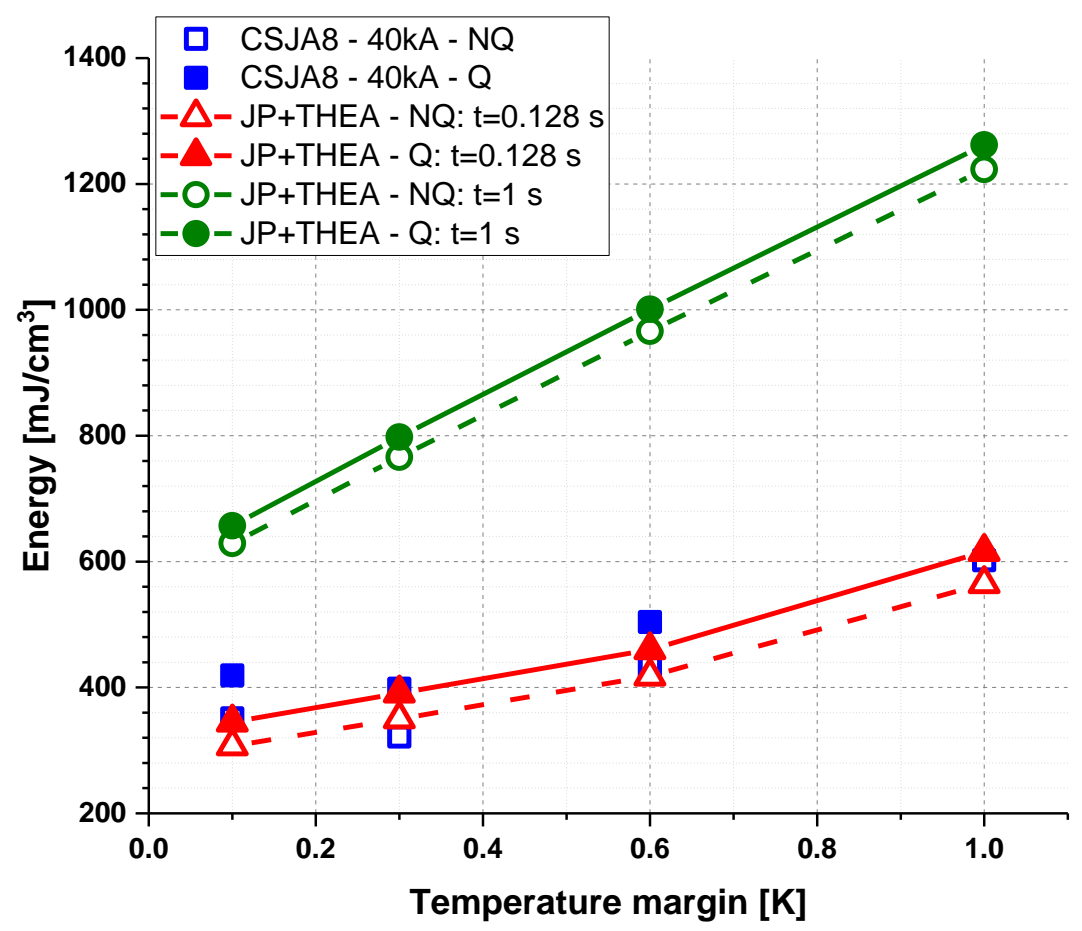

Figure 3 - Stability test of the CSJA8 conductor with a transport current of $40 \mathrm{kA}$ and a magnetic field pulse period of 0.128 and $1 \mathrm{~s}$. The energy, measured in Sultan and calculated with JackPot-THEA, is shown as function of the temperature margin. The square symbols are the experimental data from the SULTAN tests, the lines are the energy values calculated with JackPot-THEA. Open symbols and dash lines indicate recovery, filled symbols and solid lines indicate a quench.

The temperature profiles of the no-quench and quench simulations for 0.128 and $1 \mathrm{~s}$ pulses are shown in Figure 4 and Figure 5, the temperature is calculated in the HFZ. For both simulations the initial temperature is $T_{\text {in }}=8.1 \mathrm{~K}$. The quench starting moment is different for the two pulses. For $0.128 \mathrm{~s}$ pulse period, it coincides with the second power deposition peak that corresponds to the maximum $d B / d t$ of the sinusoid pulse field. While for $1 \mathrm{~s}$ pulse the third peak triggers the quench. The helium, due to the longer pulse duration, extracts more heat from the cable allowing a higher MQE for the $1 \mathrm{~s}$ pulse. Before quenching, the conductor can reach more than $10 \mathrm{~K}$ in both cases, while the helium temperature reaches $8.7 \mathrm{~K}$ during the fast pulse deposition and $9.8 \mathrm{~K}$ with $1 \mathrm{~s}$ pulse. The helium has slower temperature increase than the cable, which is determined by the conductor wetted perimeter and heat transfer, the energy exchanged with the helium is directly proportional to the contact area. The wetted perimeter defines together with the helium heat transfer, the amount of heat, which can be extracted from the cable per unit of time. 


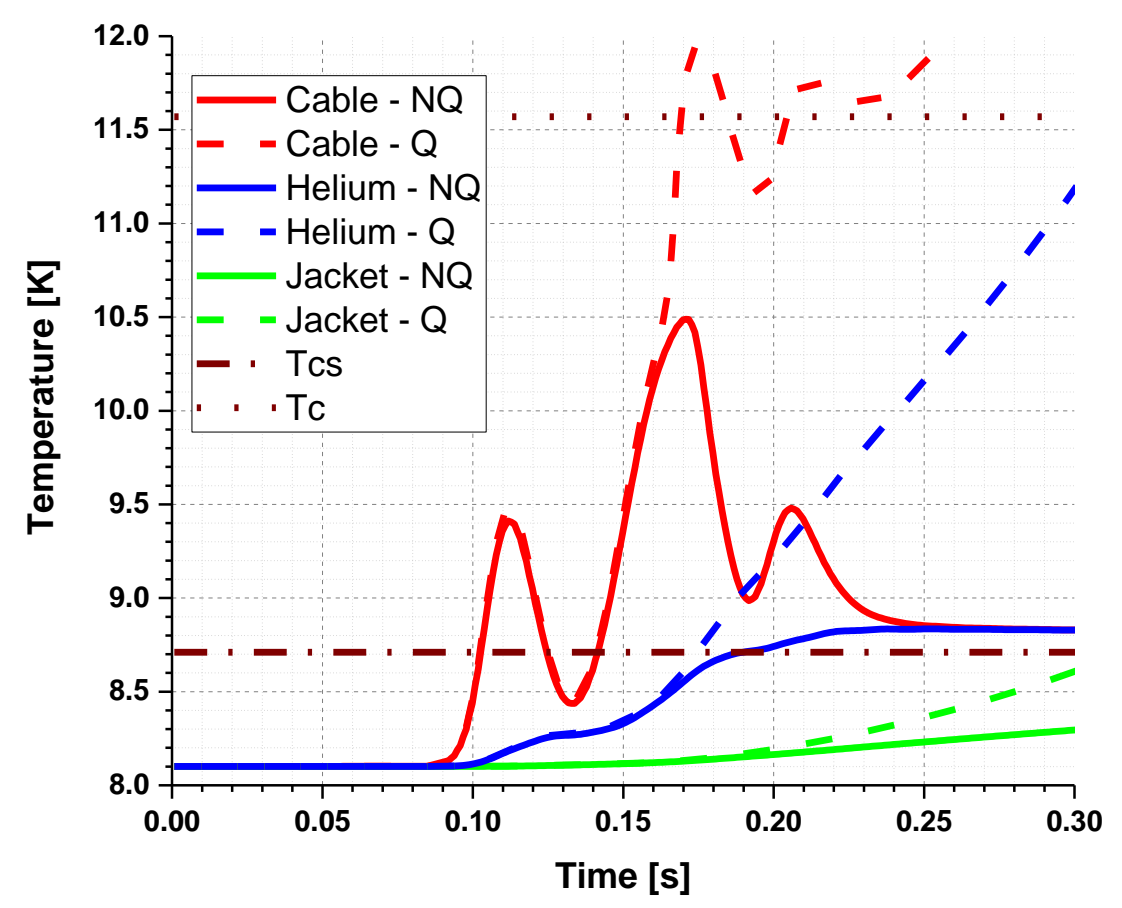

Figure 4 - CSJA8 temperature profile during JackPot-THEA stability simulations with $0.128 \mathrm{~s}$ sine wave pulse period. The solid lines represent the conductor recovery, while the dashed lines represent the temperature behavior during a quench. The horizontal dashed lines are the $T_{\mathrm{cs}}$ and the $T_{\mathrm{c}}$ of the sample.

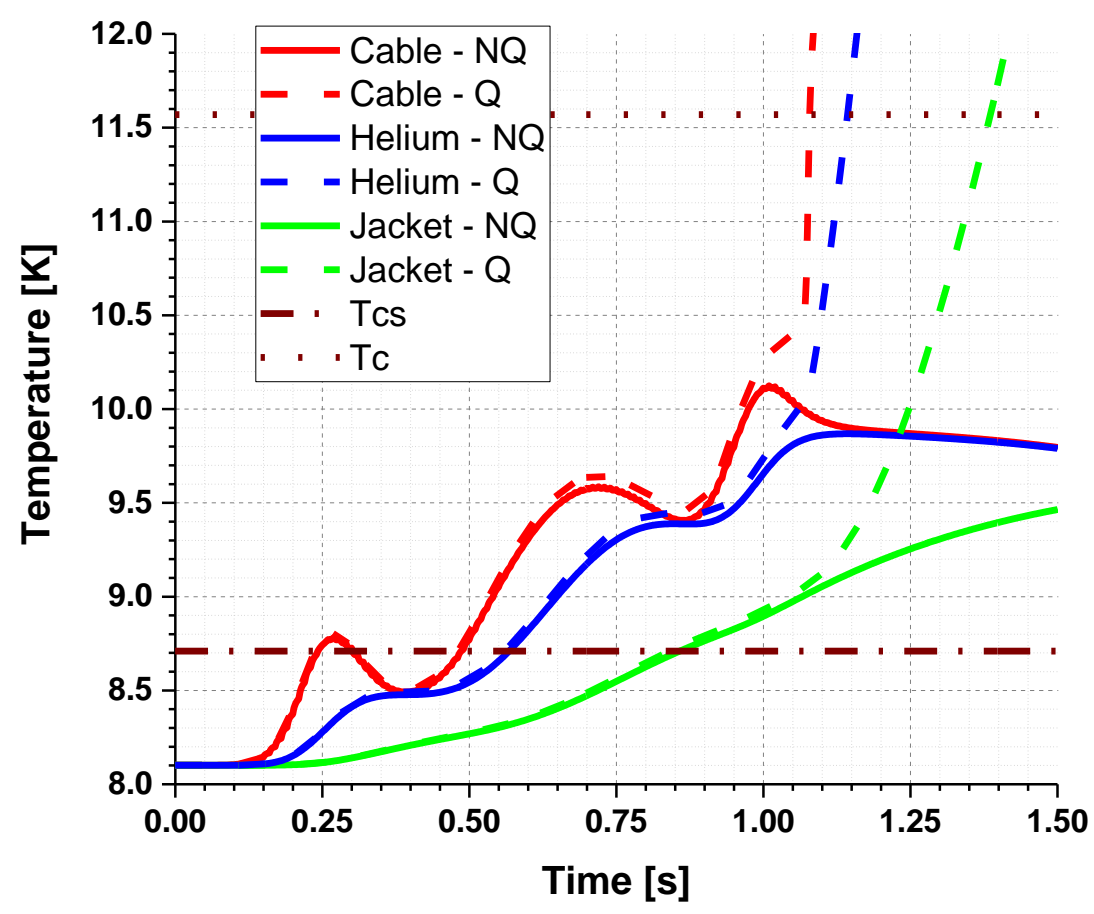

Figure 5 - CSJA8 temperature profile during JackPot-THEA stability simulation with $1 \mathrm{~s}$ sine wave pulse period. 


\section{Stability for $5 \mathrm{~s}$ pulse period}

Keeping the same approach and testing conditions as used for the $1 \mathrm{~s}$ pulse simulation, the pulse period is now increased to $5 \mathrm{~s}$. The magnetic field pulse amplitude in the simulation is progressively increased till a quench occurs in the conductor. During the pulse period of $5 \mathrm{~s}$, the helium temperature practically reaches equilibrium with the conductor; therefore both elements almost follow the same temperature profile. The temperature profile of the stability simulation in the HFZ, starting at $T_{\text {in }}=8.1$ $\mathrm{K}$, is shown in Figure 6. The conductor reaches the maximum temperature during the third power peak of the pulse and the cable quenches after a short decrease of temperature and time delay. This type of profile seems to be unrealistic at first sight, because the temperature decrease before the quench. But it is a limitation of the $2 \mathrm{D}$ visualization which does not show the effect of the helium mass flow.

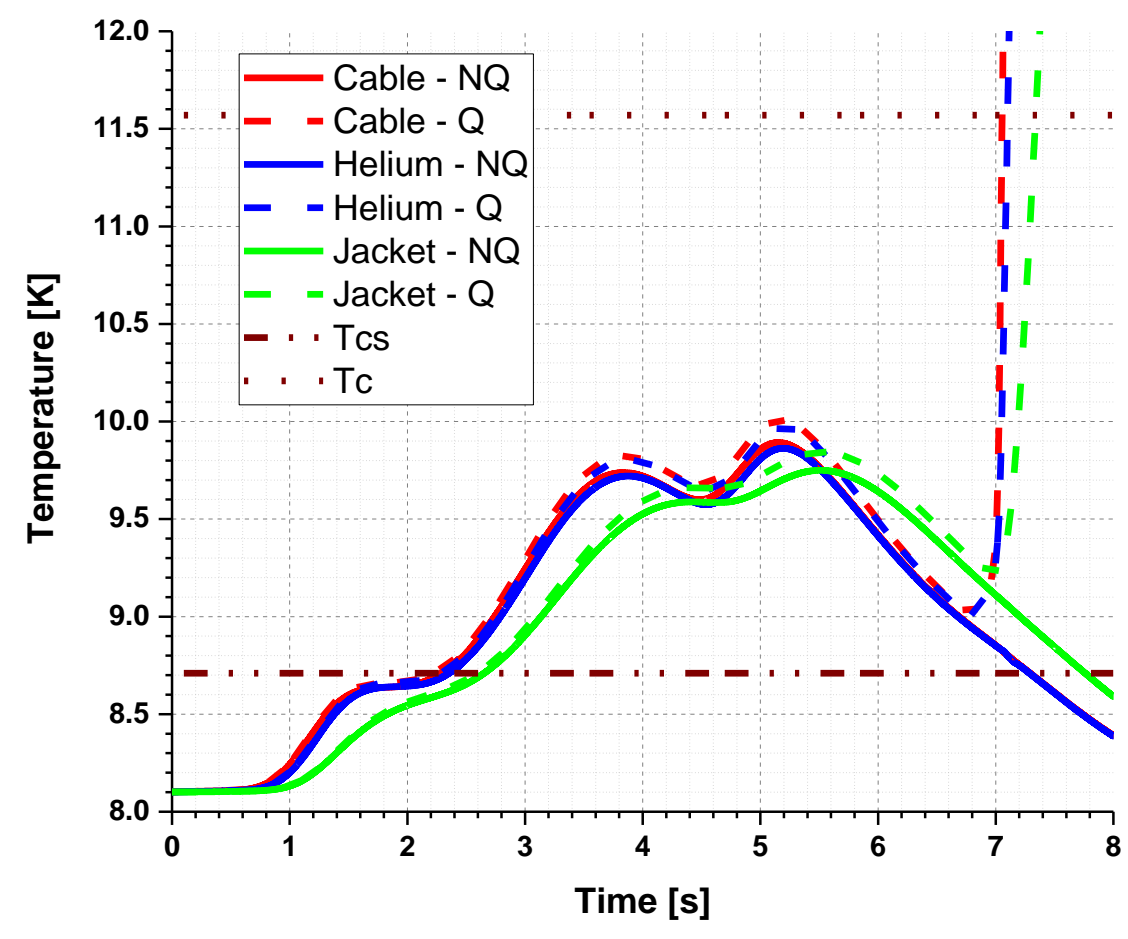

Figure 6 - CSJA8 temperature profile during the stability simulations with 5 s sine wave pulse period. The solid lines represent the conductor recovery, while the dashed lines represent the temperature behavior during a quench. The horizontal dashed lines are the Tes and the Tc of the sample.

The impact of the helium flow on the cable quench behavior can be understood from the plot of the temperature as a function of time and cable length, see Figure 7. The two black solid lines define the HFZ boundaries and the $T_{c s}$ is represented by the black dots on the surface. The helium flow direction is indicated on the graph. The temperature peak during the pulse deposition is reached at $t \sim 5 \mathrm{~s}$. The temperature progressively keeps increasing downstream the HFZ. The helium temperature is in quasiequilibrium with the self-heating of the conductor, until the quench avalanche starts, around $t \sim 6 \mathrm{~s}$ and the entire cable quenches. 


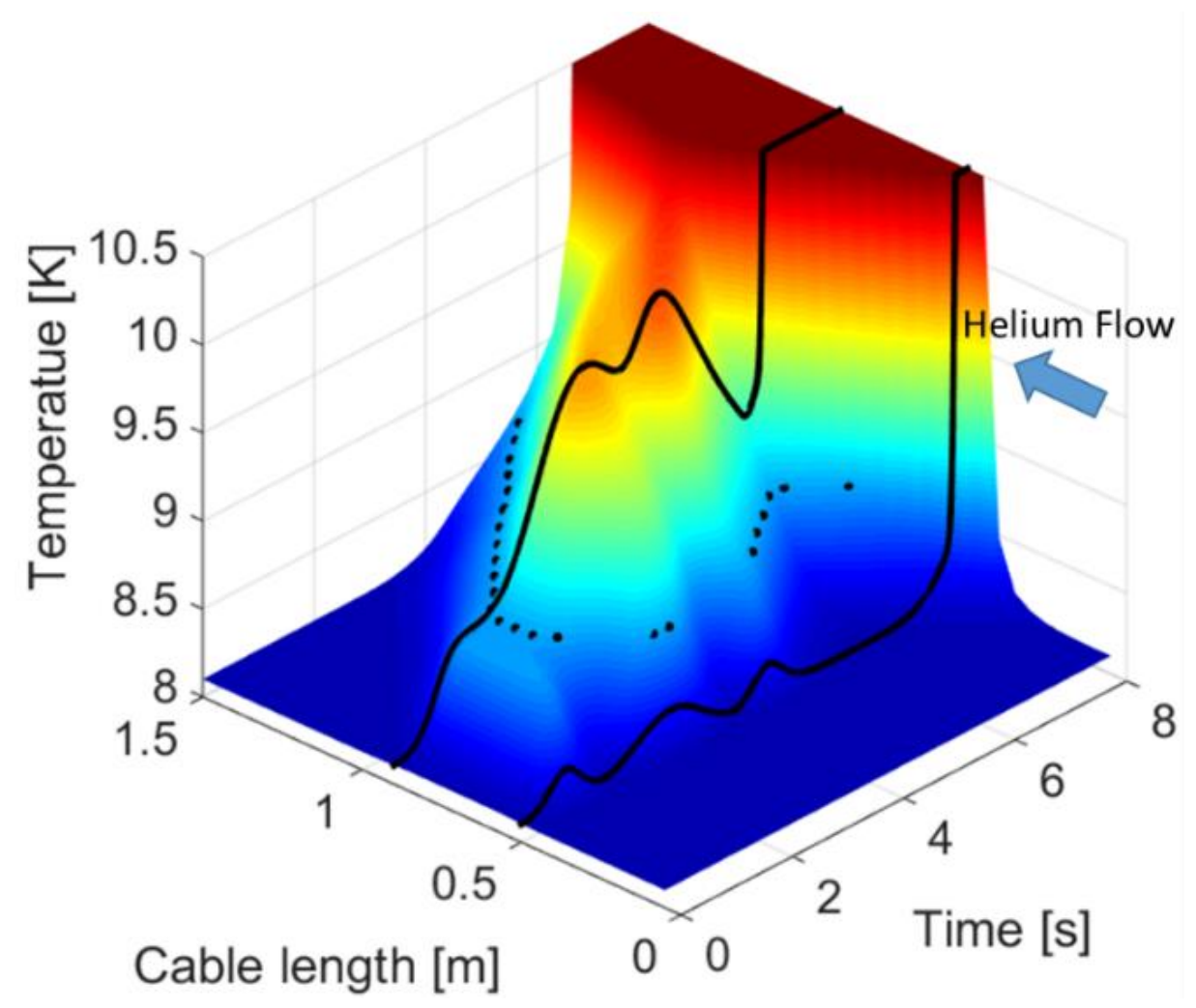

Figure 7 - CSJA8 temperature evolution as function of time and conductor length. The solid black lines define the heating zone, the black dots the $T_{\text {cs. }}$ Applied testing conditions are: $\mathrm{T}=8.1 \mathrm{~K}, \mathrm{~B}_{\mathrm{dc}}=9.7 \mathrm{~T}, \mathrm{I}_{\mathrm{op}}=40 \mathrm{kA}, \mathrm{B}_{\mathrm{a}}=2.4 \mathrm{~T}$ and $t_{\text {pulse }}=5 \mathrm{~s}$. The conductor is with blocked central channel as in the SULTAN experiment.

The results of the model in Figure 7 reproduce the SULTAN sample with the central channel blocked as for the original conductor. The blocking of the central channel has no impact on the experimental stability tests, because the heat deposition time period of $128 \mathrm{~ms}$ is too fast, as shown in [9]. In the code it is possible to implement the channel to study its effect at longer time period magnetic field pulses. The hydraulic parameters necessary to model the channel are taken from [18] and [17]. Appling the same testing conditions as used for Figure 7, i.e. same heat deposition profile, the sample with open channel will be able to recover. The channel increases the MQE limit improving the stability of the conductor in the case of longer time period sine wave pulse with lower $d B / d t$ and higher amplitude. The stability simulation results at different temperature margins with and without central channel and compared with the test at $0.128 \mathrm{~s}$ pulse period, are shown in Figure 8 . The increase of the MQE by unblocking the central channel is about $10 \%$ of the total energy deposited in the conductor. The MQE for the $5 \mathrm{~s}$ pulse period is 3.4 times larger compared with the MQE of the $0.128 \mathrm{~s}$ pulse period. In this case the pulse period ratio is $\sim 39$. 


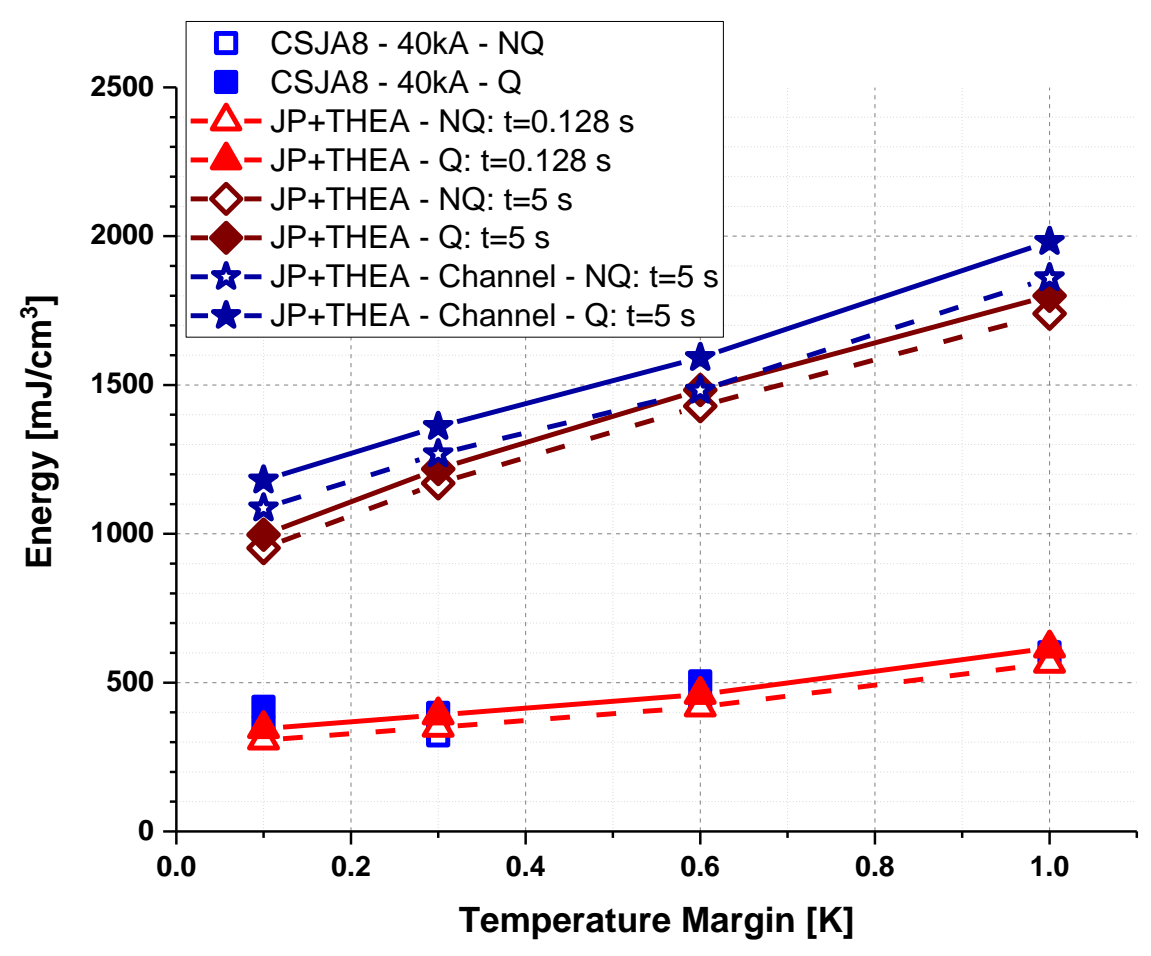

Figure 8 - Stability test of the CSJA8 conductor as function of the temperature margin between the $T_{c s}$ and the operating temperature of the test. The square symbols are the experimental data from the SULTAN tests, the lines are the energy values calculated with JackPot-THEA. Open symbols and dash lines indicate recovery, full symbols and solid lines indicate a quench.

The comparison between the average power and the energy dissipated in the CSJA8 sample during the JackPot-THEA stability simulation with different perturbation pulse periods and temperature margins is shown in Figure 9. Since the power profile is sinusoidal, the average power gives an indication of the power deposition during the stability tests. The power has an opposite trend compared with the energy. Longer sinusoidal pulse perturbation period corresponds to a higher MQE, but the average power deposited during the perturbation is lower. To reach a quench in $5 \mathrm{~s}$ of heating at $0.1 \mathrm{~K}$ of margin, it is sufficient to deposit about $200 \mathrm{~W} / \mathrm{cm}^{3}$, whereas with a perturbation of $0.128 \mathrm{~s}$ an amount of $2500 \mathrm{~W} / \mathrm{cm}^{3}$ would be required. 


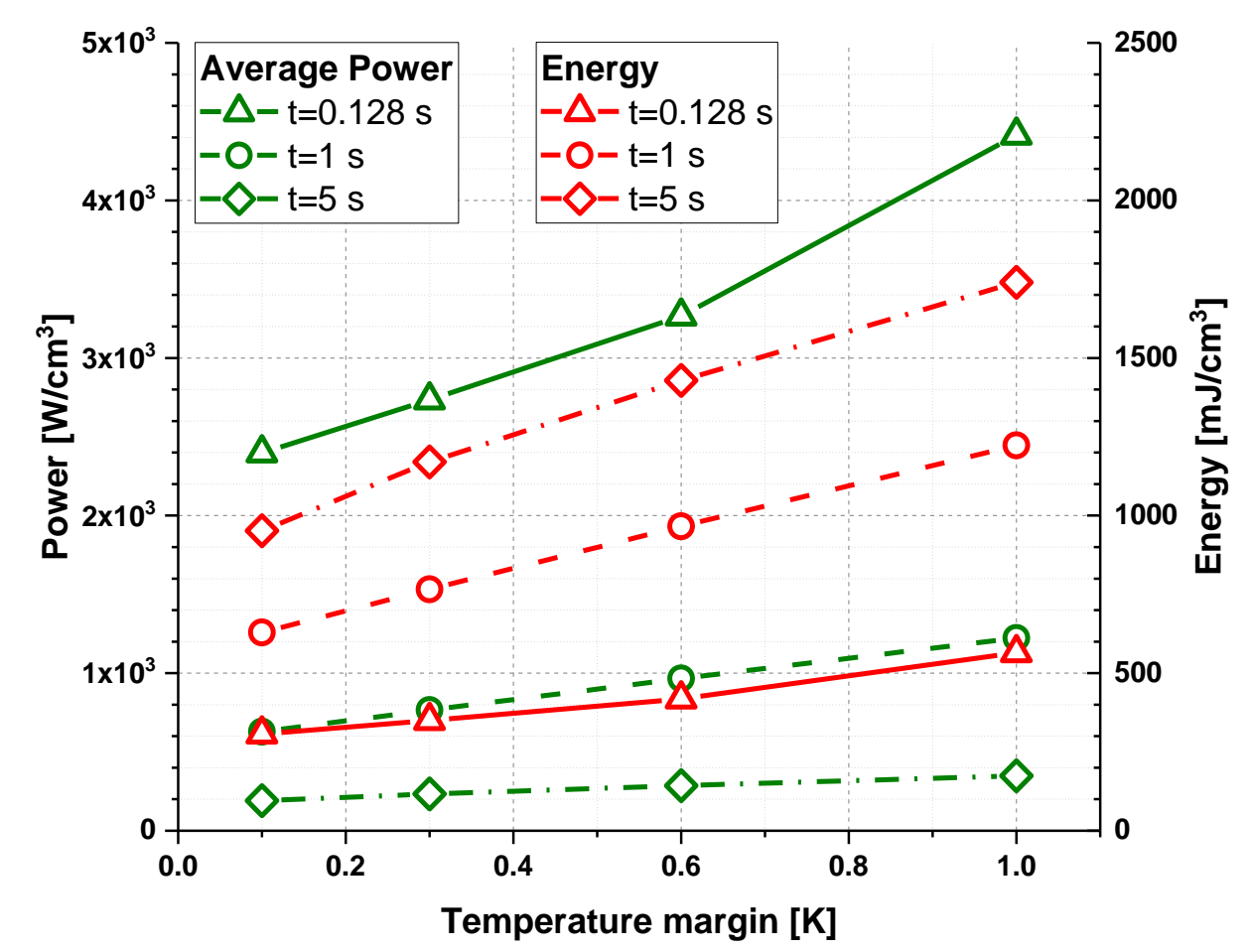

Figure 9 - Comparison of average dissipated power and energy in sample CSJA8 from the JackPot-THEA simulations at different pulse periods and temperature margins.

\section{Discussion}

A clear tendency is observed for the MQE as a function of the pulse time period, see Figure 9. The relation between MQE and the magnetic field pulse period can be expressed as:

$$
M Q E=M Q E_{\text {ref }} \cdot\left(\frac{t_{\text {pulse }}}{t_{\text {ref }}}\right)^{\frac{1}{3}},
$$

where the MQE is proportional to the cubic root of the ratio between the magnetic field pulse periods. Considering the experimental MQE at $t_{\text {pulse }}=0.128 \mathrm{~s}$ as reference value, the modeled MQEs can be analytically calculated. The MQE and pulse periods for the $8.1 \mathrm{~K}$ simulations and their ratio are listed in Table 5.

The energies, calculated using eq. (1), are similar to the JackPot-THEA MQE within 15\% of error, see Figure 10. The eq. (1) is valid only considering that the background field, the initial temperature, the mass flow and the pulse shape are all constant and by varying only the magnetic pulse period and its amplitude. This very simple approximation could be useful to have a rough estimation of the MQE generated by different perturbation periods. However, it does not give any information on the magnetic field amplitude necessary to reach the MQE.

Table 5 - Summary of the magnetic field pulse periods and their MQE calculated at $8.1 \mathrm{~K}$ and their relative ratio's with the reference SULTAN pulse and $M Q E$, $t_{\text {ref }}=0.128 \mathrm{~s}$ and $\mathrm{MQE}_{\text {ref }}=418 \mathrm{~mJ} / \mathrm{cm}^{3}$ at $T=8.1 \mathrm{~K}$.

\begin{tabular}{|c|c|c|c|c|}
\hline & $t_{\text {pulse }} / t_{\text {ref }}$ & $\begin{array}{l}M Q E \\
(T=8.1 \mathrm{~K})\end{array}$ & $M Q E / M Q E_{r e f}$ & $\begin{array}{l}M Q E \\
\text { eq.(1) }\end{array}$ \\
\hline$t_{l}=1 \mathrm{~s}$ & 7.81 & 966 & 2.3 & 829 \\
\hline$t_{2}=5 \mathrm{~s}$ & 39 & 1429 & 3.4 & 1418 \\
\hline
\end{tabular}




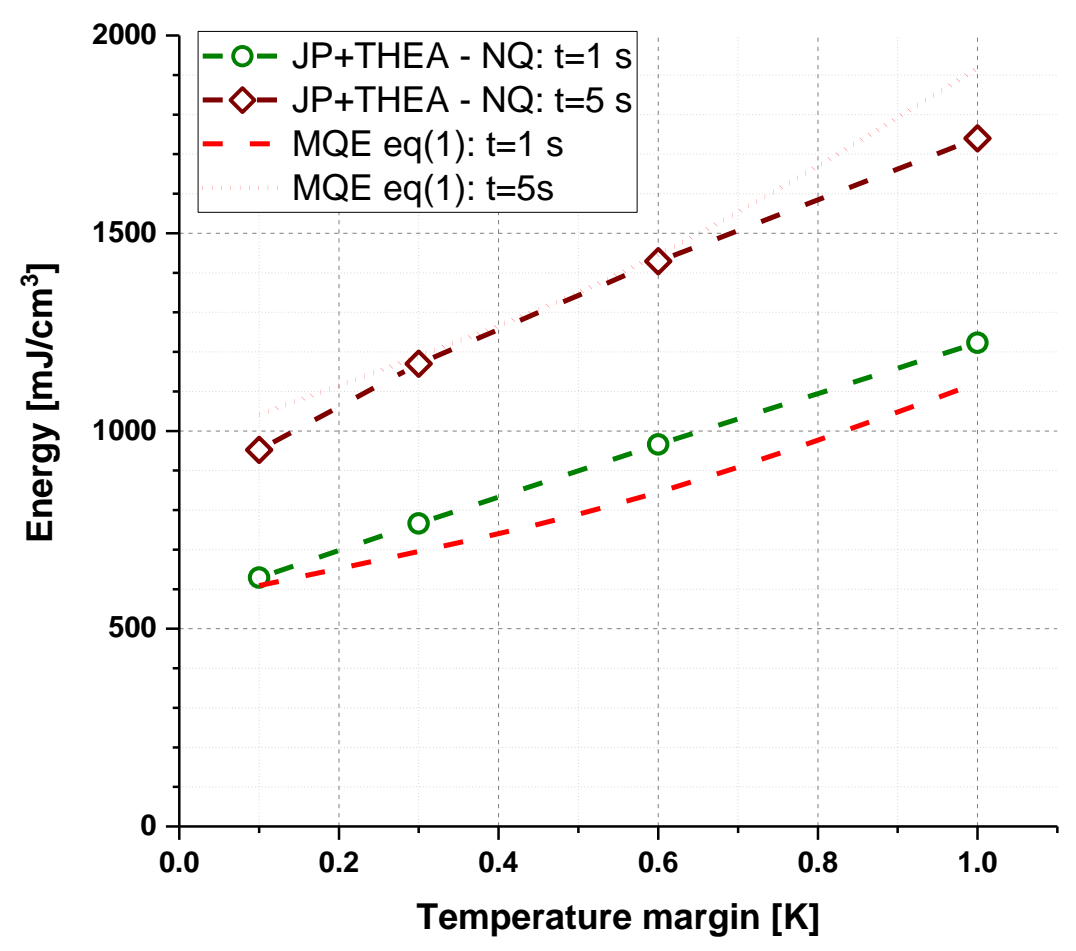

Figure 10 - Comparison between the MQE simulated with JackPot-THEA at different pulse periods and the MQE calculated with eq. (1).

Looking at the quench temperatures $\left(T_{Q}\right)$, see Figure 4, Figure 5 and Figure 6 , it can be observed that $T_{Q}$ decreases slowly with the increase of the single pulse period from $10.5 \mathrm{~K}$ for $t_{p u l s e}=0.128 \mathrm{~s}$ to 10 $\mathrm{K}$ for $t_{\text {pulse }}=5 \mathrm{~s}$. For longer perturbations able to generate a quench, the $T_{Q}$ decreases but still holds $T_{Q}$ $>T_{c s}$. The coolant flow, kept at the test initial temperature, removes part of the deposited heat from the cable, allowing the conductor to handle temperatures higher than $T_{c s}$ for a short period of time.

A similar behavior is noted analyzing the electric field of the conductor. JackPot calculates the local electric field from the current and critical current distribution in the strands, as [19]:

$$
E=E_{c}\left(\frac{I_{s t r}}{I_{c}}\right)^{n}
$$

Knowing when the quench starts, it is possible to calculate the peak electric field $\left(E_{Q}\right)$ for the MQE and the average electric field $\left(E_{\text {avg }}\right)$. Since the coupling current is proportional to $(d B / d t)^{2}$, the power dissipation and the electric field are inversely proportional to the pulse period. Therefore, the electric field at the MQE is lower for longer pulse periods. This is also related to the effective temperature of the helium during the deposition. For fast deposition, see Figure 4, the helium temperature is lower than the temperature of the conductor during the pulse. This allows the conductor to reach a very high peak electric field and yet being able to recover after the end of the pulse. Whereas for longer pulses, see Figure 5 and Figure 6, the temperature gradient between the helium and the conductor decreases and the conductor is not able to recover even if the peak electric field is lower than at short pulse.

The peak and the average of the electric fields as a function of the pulse period and temperature margin are shown in Figure 11 and Figure 12, respectively. The $E_{Q}$ is compared with the peak electric 
field calculated from the SULTAN fast pulse tests, whereas the $E_{\text {avg }}$ with the average electric field measured during the $T_{c s}$ tests on different CS samples [20]. When the magnetic field pulse period of $0.128 \mathrm{~s}$ is applied in the temperature margin range $T_{\text {margin }}=0.1-1 \mathrm{~K}$, the simulated peak electric field is about $E_{\mathrm{Q}} \sim 200-300 \mathrm{mV} / \mathrm{m}$, while the average electric field is $E_{\text {avg }} \sim 5-8 \mathrm{mV} / \mathrm{m}$. The $E_{Q}$ and $E_{\text {avg }}$ are respectively 4 and 2 orders of magnitude larger than $E_{c}(10 \mu \mathrm{V} / \mathrm{m})$. Increasing the pulse period, the electric fields decrease and $E_{\text {avg }}$ reaches a value of about $450 \mu \mathrm{V} / \mathrm{m}$ at $t_{\text {pulse }}=5 \mathrm{~s}$. This value is comparable with the average electric field, measured in steady state conditions on CS conductors during the $T_{c s}$ tests performed in SULTAN, which is about $200-400 \mu \mathrm{V} / \mathrm{m}$. Therefore, increasing the magnetic field pulse duration, the simulated average electric field approaches the experimental critical electric field measured in DC condition, proving that the extrapolations tendency made with JackPotTHEA is in good agreement with the SULTAN experimental results and can be used to predict the behavior of CiCCs and improve their design.

The critical electric field measured in DC condition can be used to analyze the limits of DC magnets in both cases where the magnetic field is constant or has very long fluctuation periods. On the other hand, in AC magnets the conductors show the ability to cross the $E_{c}$ limit without quenching. From the simulation results, we can argue that the quench occurs after reaching a certain threshold of electric field. Consequently, $E_{Q}$ and $E_{\text {avg }}$ can be used as quantitative AC threshold for this type of conductors.

In practical terms, taking as example the ITER Central Solenoid, it is possible to use JackPot to calculate the peak electric field during the plasma scenario. If the calculated values are comparable or higher than the electric field threshold, the CS conductor will operate in critical condition. In other words, the CS conductor will operate in optimal condition if its peak electric field is lower than the threshold. Another possible application might be for future conductor designs since knowing the operating condition of the conductor would allow simulating the electric field and determining possible issues improving the design of the conductors. 


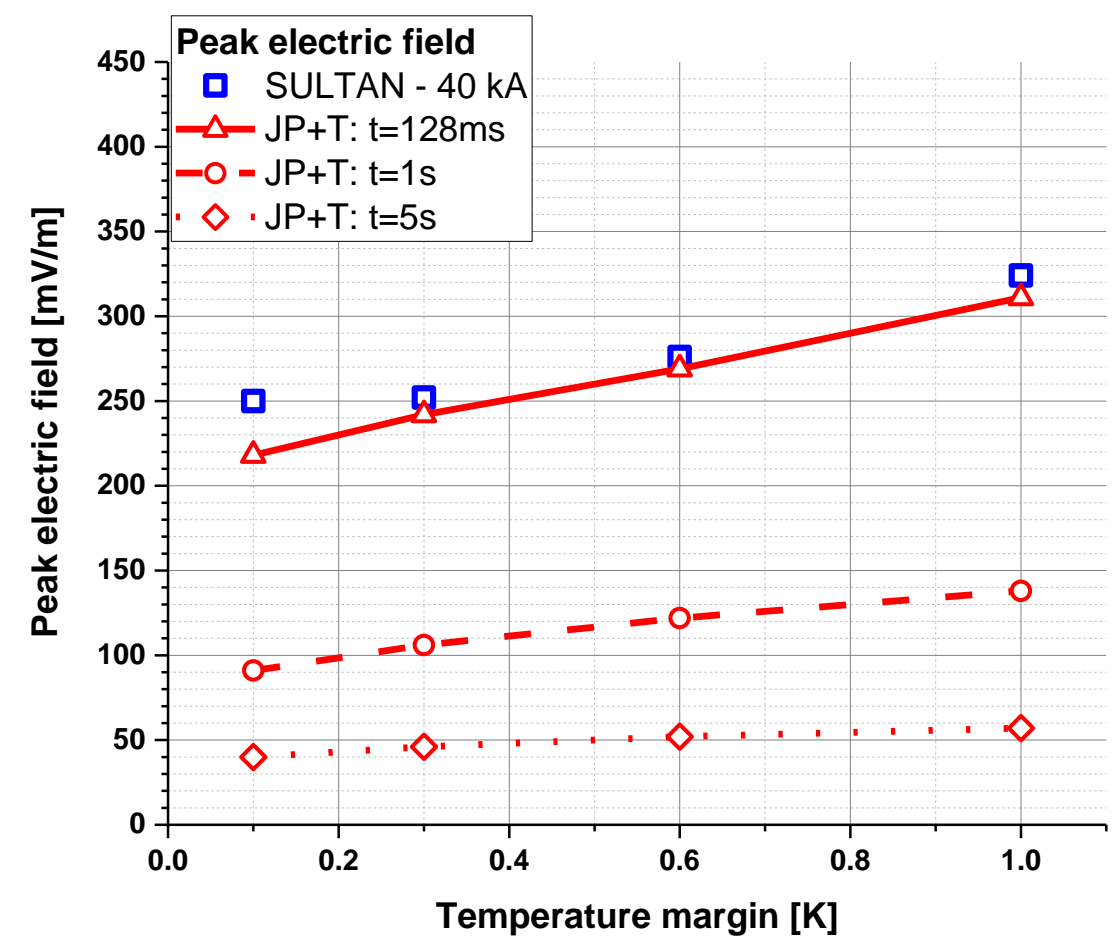

Figure 11 - The CSJA8 peak electric field in function of the temperature margin and pulse period, simulated with JackPot-THEA, is compared with the peak electric field calculated from the SULTAN stability test. In all the simulations $B_{\mathrm{dc}}=9 \mathrm{~T}$ and $I=40 \mathrm{kA}$.

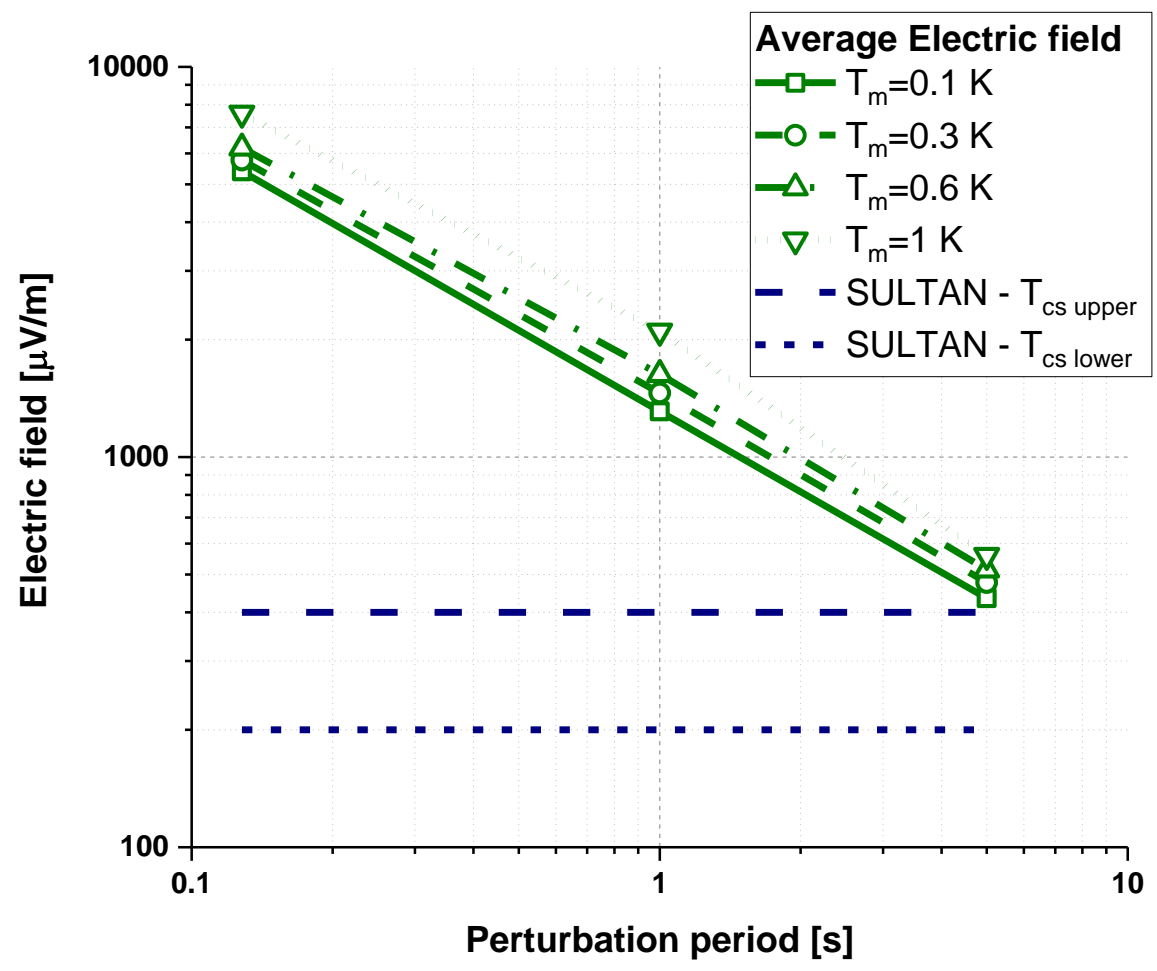

Figure 12 - The average electric field in function of the perturbation period and the temperature margin of the sample CSJA8 simulated with JackPot-THEA and compared with the quench electric field measured in SULTAN $T_{c s}$ tests performed on ITER CS samples [20]. In all the simulations $B_{\mathrm{dc}}=9 \mathrm{~T}$ and $I=40 \mathrm{kA}$. 


\section{Conclusions}

The JackPot model calculates accurately the energy deposited inside a cable for any time dependent applied magnetic field, current and temperature. The combination JackPot-THEA is able to predict the SULTAN MQE experiment for the ITER CSJA8 sample with excellent agreement. This implies that JackPot-THEA provides a good basis for stability analyses of ITER coils subjected to severe alternating magnetic fields like during the plasma operating scenario.

The JackPot-THEA extrapolated results towards longer pulse duration show the dependency of the MQE with the sinusoidal magnetic field pulse period. The MQE becomes larger with increasing magnetic field pulse period. The average power deposition to reach a quench is inversely proportional to the power deposition time.

The $5 \mathrm{~s}$ slow pulse simulation shows the influence of the central channel on the stability. Unblocking the central channel increases the MQE margin by about $10 \%$.

The peak electric field at the MQE is investigated using the results from the JackPot-THEA simulations with different pulse periods. The peak electric field can be used as an electromagnetic threshold for modeling CICCs. The $E_{Q}$ and $E_{\text {avg }}$ thresholds are significantly higher than the quench electric field measured in DC condition. However, the $E_{\text {avg }}$ approaches the experimental steady-state quench electric field when increasing the magnetic field pulse period.

The extrapolation of stability test simulations, validated by $0.128 \mathrm{~s}$ pulse duration SULTAN measurements, to the $5 \mathrm{~s}$ range by using JackPot-THEA, opens the possibility to an electromagnetic and thermal stability comparison between the short-sample stability tests and the ITER Plasma Scenario conditions.

\section{References}

[1] T. Kato, "First test results for the ITER central solenoid model coil", Fusion Engineering and Design, vol. 56-57, pp. 59-70, 2001.

[2] P. Bruzzone, A. Anchel, A. Fuchs, G. Pasztor, B. Stepanov, M. Vogel and G. Vecsey, "Upgrade of Operating Range for SULTAN Test Facility", IEEE Transaction on Applied Superconductivity, vol. 12, no. 1, pp. 520-3, 2002.

[3] A. Devred and Et al., "Conductor qualification program for the ITER central solenoid", IEEE Trans. Appl. Supercond., vol. 23, no. 3, p. 6001208, 2013.

[4] E.P.A. van Lanen and A. Nijhuis, "JackPot: A novel model to study the influence of current nonuniformity and cabling patterns in cable-in-conduit conductor", Cryogenics, vol. 50, no. 3, pp. 139-148, 2010.

[5] E.P.A. van Lanen and A. Nijhuis, "Simulation of interstrand coupling loss in cable-in-conduit conductors with JackPot-AC", IEEE Transaction on Applied Superconductivity, vol. 21, no. 3, pp. 1926-1929, 2011. 
[6] E.P.A. van Lanen, J. van Nugteren and A. Nijhuis, "Validation of a strand-level CICC-joint coupling loss model", Superconductor Science and Technology, vol. 25, no. 2, p. 025013, 2012.

[7] L. Bottura, C. Rosso and M. Breschi, "A General Model for Thermal, Hydraulic and Electric Analysis of Superconducting Cables", Cryogenics, vol. 40, no. 8-10, pp. 617-626, 2000.

[8] L. Bottura and M. Marinucci. http://www.htess.com/cryosoft.htm.

[9] T. Bagni, M. Breschi, J.L. Duchateau, A. Devred and A. Nijhuis, "Analysis of ITER Nb-Ti and Nb3Sn CICCs experimental Minimum Quench Energy with JackPot, MCM and THEA models", Superconductor Science and Technology, vol. 30, no. 9, p. 095003, 2017.

[10] N. Mitchell, "Scenarios for coil, power supply and cryoplant analysis v1.10", ITER-IO, Technical report, 2FTVKY, 2009.

[11] B. Stepanov, P. Bruzzone, R. Wesche, N. Martovetsky, D. Hatfield, A. Vostner and A. Devred, "Impact of Sample Preparation Procedure on the Test Results of Four US ITER TF Conductors", IEEE Transaction on Applied Superconductivity, vol. 20, no. 3, pp. 508 - 511, 2010.

[12] P. Bruzzone, A. Fuchs, B. Stepanov and G. Vecsey, "Performance evolution of Nb3Sn cable-inconduit conductors under cyclic load [for Tokamaks]", IEEE Transaction on Applied Superconductivity, vol. 12, no. 1, pp. 516-9, 2002.

[13] L. Bottura and B. Bordini, "Jc(B,T) Parametrization for the ITER Nb3Sn Production", IEEE Trans. Appl. Supercond., vol. 19, no. 3, pp. 1521-1524, 2009.

[14] S.P. Kwon, K. Sim, Y. J. Ma, S. Park, P. Park, I. Shin, H. Choi, Y. Seo, K. Jang and W. Park, "Prototype Nb3Sn Superconducting Strad for ITER CS Coil Conductor Produced in Korea Using the Internal-Tin Route", IEEE Transactions on Applied Supercond., vol. 25, no. 3, p. 4201106, 2015.

[15] A. Nijhuis and et al., "The effect of axial and transverse loading on the transport properties of ITER Nb3Sn strands", Superconductor Science and Technology, vol. 26, no. 8, p. 084004, 2013.

[16] M. Bagnasco, "Calorimentric Method for current sharing temperature measurements in ITER conductor samples in SULTAN", Fusion Engineering and Design, vol. 84, no. 2-6, pp. 423-426, 2009.

[17] D. Bessette, "Conductor Parameters for 2008-2009 analyses", ITER-IO, Internal report, 2009.

[18] N. Mitchell, "Design Description Document: 11 - Magnet, ITER_D_22HV5L v2.2", ITER-IO, Technical report, 2009.

[19] L. Bottura, "Cable Stability", in Proceedings of CERN Accelerator School, 2013.

[20] G. Rolando, A. Devred and A. Nijuis, "Temperature and current margin of ITER central solenoid conductor designs during a 15MA plasma scenario", Superconductor Science and Technology, 
vol. 27, no. 2, p. 025010, 2014. 